\title{
The Association between Daily Total Dietary Nutrient Intake and Recent Glycemic Control States of Non-Pregnant Adults 20+ Years Old from NHANES 1999-2018 (Except for 2003-2004)
}

\author{
Yin Bai ${ }^{1}$, Hao Zhang ${ }^{2}$, Jie Yang ${ }^{3}$ and Lei Peng ${ }^{4, *(D)}$ \\ 1 Department of Marketing, College of Management and Economics, Tianjin University, Tianjin 300072, China; \\ baiyin@tju.edu.cn \\ 2 Department of Social Medicine and Health Education, School of Public Health, Peking University, \\ Beijing 100000, China; 2011210118@stu.pku.edu.cn \\ 3 Public Health Clinical Center of Chengdu, Chengdu 610066, China; yangjieHJ@gmail.com \\ 4 Department of Epidemiology, School of Clinical Oncology, Peking University, Beijing 100143, China \\ * Correspondence: 2011210593@stu.pku.edu.cn; Tel.: +86-138-0828-2849
}

check for updates

Citation: Bai, Y.; Zhang, H.; Yang, J.; Peng, L. The Association between Daily Total Dietary Nutrient Intake and Recent Glycemic Control States of Non-Pregnant Adults 20+ Years Old from NHANES 1999-2018 (Except for 2003-2004). Nutrients 2021, 13, 4168. https://doi.org/10.3390/ nu13114168

Academic Editor: Lourdes Varela

Received: 18 September 2021

Accepted: 19 November 2021

Published: 21 November 2021

Publisher's Note: MDPI stays neutral with regard to jurisdictional claims in published maps and institutional affiliations.

Copyright: (c) 2021 by the authors. Licensee MDPI, Basel, Switzerland. This article is an open access article distributed under the terms and conditions of the Creative Commons Attribution (CC BY) license (https:/ / creativecommons.org/licenses/by/ $4.0 /)$.

\begin{abstract}
Background: Although daily total dietary nutrient intakes were potentially important factors in maintaining glycemic balance, their overall effect on glycemic control was still unclear among American adults. Objectives: We aimed to examine the association between daily total dietary nutrient intake and recent glycemic control status (RGCS). Methods: This cohort was composed of 41,302 individuals from the National Health and Nutrition Examination Survey (NHANES). The daily total intake of dietary nutrients and RGCS were independent and dependent variables, respectively. To evaluate their association, we carried out binary logistic regression, model fitting, linear discriminant analysis, and the receiver operator characteristic (ROC) analysis. Results: The result of robust check model showed that only the daily total dietary vitamin B6 intake (adjusted OR $=0.848$; 95\% CI: 0.738, 0.973; $p$-value $=0.019$ ) was significantly negatively correlated with RGCS. When daily total dietary vitamin $\mathrm{B} 6$ intake and glycosylated hemoglobin $(\mathrm{HbA} 1 \mathrm{c})$ were used as independent variables and dependent variables, respectively, to fit the curves and lines, the established robust check model could distinguish American adults with different RGCS well. Moreover, the robust check model results of ROC analysis indicated that daily total dietary vitamin B6 intake might be a potential predictor for RGCS (AUC $=0.977 ; 95 \%$ CI: 0.974, 0.980; $p$-value < 0.001). Conclusions: This study showed that only daily total dietary vitamin B6 intake was a beneficial factor in RGCS, but it might need further multicenter or prospective studies to verify whether vitamin B6 had biological implications and public health meaning for glycemic control among American adults (specifically referred to non-pregnant participants over 20 years old).
\end{abstract}

Keywords: daily total intake of dietary nutrients; RGCS; HbA1c; NHANES; odds ratio

\section{Introduction}

Dietary nutrients play an important role in maintaining the balance of blood glucose as a necessary substance to regulate the normal physiological function of the body, being roughly divided into macronutrients [1-3], dietary fiber (as an independent factor that distinguishes carbohydrates, included in this study) [4-6], minerals [7-9], and vitamins [10-13]. Many studies have shown that there was a positive correlation between recent glycemic control status (RGCS) and serum chromium, zinc, and magnesium levels [7,14-20]. Glycosylated hemoglobin (HbA1c), an irreversible product of blood glucose and hemoglobin, could provide information for long-term glycemic control [21]. Moreover, after the relationship between RGCS and HbA1c concentration was widely confirmed, the serum index was applied to diabetes diagnosis and glycemic monitoring practice [21,22]. Therefore, it was appropriate to use HbA1c as a predictor for RGCS among American adults (specifically referred to non-pregnant participants over 20 years old). 
At present, most of the research conclusions on the association between daily total nutrient intake and RGCS have been one-sided. They did not analyze the overall effect of various nutrients on RGCS but analyzed minerals, vitamins, and macronutrients separately, which was not complete and systematic, and might have even led to obtaining inconsistent conclusions $[23,24]$. Although findings on the association between daily total dietary nutrient intake and RGCS were inconsistent and not enough to prove the relationship, these results, to a certain extent, could supply research hypotheses for future large-scale prospective or multi-center verification. Therefore, if we further explored the association between the adults' RGCS and daily total dietary nutrient intakes, it was necessary to construct a holistic and optimal model to combine the macronutrients, minerals, and vitamins of daily total nutrient intake, as well as demographic characteristics, in order to draw a reliable conclusion.

In addition, most studies on dietary factors affecting glycemic control have been conducted on diabetes patients [5,11,25-27], and therefore these conclusions could not be suitable for American adults to control blood glucose. Moreover, it is worth noting that insufficient sample size might also lead to biased conclusions, for instance, in the study of Intra et al. [26], the sample size of cases group was very small (cases group, $n=84$; controls group, $n=2745$ ), and therefore the results of this study might have a larger systematic bias. Therefore, we conducted the follow-up sampling survey study to estimate the association between daily total nutrient intake and RGCS among non-pregnant adults 20+ years old using a large-scale database from National Health and Nutrition Examination Survey (NHANES 1999-2018, except for 2003-2004).

\section{Methods}

\subsection{Database and Study Population}

We used the NHANES database, a nationally representative survey database collected biennially by the National Center for Health Statistics (NCHS), and employed a complex, multistage, probabilistic sampling design [28]. The database was publicly available on the Internet and can be downloaded by researchers around the world. All details about the database could be efficiently acquired at http:/ / www.cdc.gov/nchs/nhanes/ (accessed on 27 May 2021), including relevant information such as strict quality control measures for the questionnaire data undertaken by NHANES. The $24 \mathrm{~h}$ dietary recall data from non-pregnant adults 20+ years of age participating in NHANES 1999-2002 and 2005-2018 surveys were followed biennially for all analyses. The database for analysis consisted of five parts: demographics data, dietary data, examination data, laboratory data, and questionnaire data.

During the 1999-2018 NHANES survey cohorts, 101,316 preliminary participants were included in the study. Individuals without physical examination data $(n=2096)$, under 20 years of age $(n=47,208)$, pregnant $(n=2527)$, without an unusual diet compared food consumed yesterday and without reliable data $(n=6431)$, and 2003-2004 survey cycle data with the missing outcome variable $(n=1752)$ were excluded. Those with complete or reliable $24 \mathrm{~h}$ recall data (only day 1 data used) as judged by the United States Department of Agriculture's (USDA) Food Surveys Research Group staff were included in the analyses. In addition, of the 41302 participants, 58 of the dietary survey data contained some missing indices (such as the intake of dietary fiber and folic acid), and we used the median to fill them. Finally, 41,302 subjects (20,458 males and 20,844 females, $50.0 \pm 17.9$ years for males and $50.2 \pm 17.8$ years for females) were certainly included in this study (Figure S1). All serum samples were collected under fasting condition. HCHS obtained the written informed consent from all participants and the ethical review committee approved all NHANES protocols.

\subsection{Variables}

In this study, the independent variables were daily total dietary nutrient intakes, containing protein, carbohydrate, total fat, dietary fiber, vitamin B1, vitamin B2, vitamin 
B6, total folate, vitamin B12, vitamin E, calcium, magnesium, iron, zinc, and copper. It is worth mentioning that the dietary energy value different from the category of dietary nutrients was also included in the follow-up analysis as an independent important parameter. The dependent variable was RGCS ( $\mathrm{HbA} 1 \mathrm{c}<6.5 \%$ represents good RGCS, and $\mathrm{HbA} 1 \mathrm{c} \geq 6.5 \%$ represents poor RGCS). All variables involved in this study were divided into continuous variables and categorical variables. Continuous variables included energy, protein, carbohydrate, dietary fiber, total fat, total folate, vitamin B1, vitamin B2, vitamin B6, vitamin B12, vitamin E, calcium, magnesium, iron, zinc, copper, poverty income ratio (PIR), insulin, glucose, and hemoglobin. Categorical variables included gender, age, race, education level, body mass index (BMI), moderate or severe physical activity, hypertension, the doctor informing them they had diabetes, having at least 12 cups of alcoholic drink per year, consuming over 100 cigarettes in their lifetime, and adult food security. Details of all variable acquisition procedures can be found at http:/ /www.cdc.gov/nchs/nhanes/.

\subsection{Statistical Analysis}

The results of normality test showed that it could not be considered that all continuous variables obeyed normal distribution. Therefore, in the stages of statistical description and single variable analysis, all continuous variables and categorical variables were expressed as median ( $25 \%$ percentile- $75 \%$ percentile) and percentage (proportion), respectively. We used a nonparametric test (Mann-Whitney $U$ test) for all continuous variables that did not obey normal distribution, as well as Pearson's chi-squared test for all categorical variables. Then, in the multivariate analysis stage, we controlled different confounders and established four binary logistic regression models with the RGCS as the dependent variable to adjust the potential bias. Eventually, we performed model fitting with the $\mathrm{HbA} 1 \mathrm{c}$ index as the dependent variable, and receiver operator characteristic (ROC) analysis was performed to calculate the area under the curve (AUC). The result of the collinearity diagnosis showed that there was no collinearity (variance inflation factor, VIF $<10$ ) among the independent variables studied. Statistical significance was considered when $p$-value was below 0.05 (two-tailed). Data processing, statistical analysis, and graphic drawing were carried out with Stata version 13.1, IBM SPSS version 26.0, GraphPad Prism version 7.00, $R$ version 4.0.2 (http:/ / www.R-project.org, The R Foundation), and EmpowerStats software version 2.1 (http:/ / www.empowerstats.com, X\&Y Solutions, Inc., Boston, MA, USA).

\section{Result}

\subsection{Baseline Characteristics}

The description of demographic and medical characteristics is shown in Table 1. Among the participants, $49.5 \%(n=20,458)$ were male, $44.6 \%(n=18,404)$ were non-Hispanic White, $20.5 \%(n=8458)$ were non-Hispanic Black, and $17.3 \%(n=7153)$ were Mexican American. In addition, the statistical description of daily dietary nutrient intakes in our study showed that their distribution fluctuated over time (Figure 1). Therefore, the time effect was often a potential confusion factor, which should be placed in subsequent analysis.

Table 1. Characteristics by RGCS of non-pregnant adults 20+ years old from NHANES 1999-2018 (except for 2003-2004).

\begin{tabular}{|c|c|c|c|c|}
\hline Characteristics & $\begin{array}{c}\text { Good RGCS } \\
(\mathrm{HbA1}<6.5 \%) \\
n=36,594\end{array}$ & $\begin{array}{c}\text { Poor RGCS } \\
(\mathrm{HbA1} \geq 6.5 \%) \\
n=4708\end{array}$ & $x^{2} / Z$ Value & $p$-Value \\
\hline Gender (\%) & & & 21.006 & $<0.001$ \\
\hline Male & $17,978(87.9)$ & $2480(12.1)$ & & \\
\hline Female & $18,616(89.3)$ & $2228(10.7)$ & & \\
\hline Age $(\%)$ & & & 2119.291 & $<0.001$ \\
\hline$\geq 60$ years old & $11,414(80.1)$ & $2843(19.9)$ & & \\
\hline $40-59$ years old & $12,125(88.6)$ & $1554(11.4)$ & & \\
\hline$<40$ years old & 13,055 (97.7) & $311(2.3)$ & & \\
\hline
\end{tabular}


Table 1. Cont.

\begin{tabular}{|c|c|c|c|c|}
\hline Characteristics & $\begin{array}{c}\text { Good RGCS } \\
(\mathrm{HbA1}<6.5 \%) \\
n=36,594\end{array}$ & $\begin{array}{c}\text { Poor RGCS } \\
(\mathrm{HbA1} \geq 6.5 \%) \\
n=4708\end{array}$ & $x^{2} / Z$ Value & $p$-Value \\
\hline Race (\%) & & & 322.688 & $<0.001$ \\
\hline Non-Hispanic White & $16,857(91.6)$ & $1547(8.4)$ & & \\
\hline Non-Hispanic Black & $7201(85.1)$ & $1257(14.9)$ & & \\
\hline Mexican American & $6140(85.8)$ & $1013(14.2)$ & & \\
\hline Other Races & $6396(87.8)$ & $891(12.2)$ & & \\
\hline Education level (\%) ${ }^{\dagger}$ & & & 255.723 & $<0.001$ \\
\hline$\leq$ High School & $17,597(86.1)$ & $2845(13.9)$ & & \\
\hline College or above & $18,955(91.1)$ & $1855(8.9)$ & & \\
\hline $\mathrm{BMI}\left(\mathrm{Kg} / \mathrm{m}^{2}\right) * t$ & & & 219.213 & $<0.001$ \\
\hline$\geq 30.0$ & $102(87.9)$ & $14(12.1)$ & & \\
\hline$\overline{25} .0-29.9$ & $12,455(90.5)$ & $1304(9.5)$ & & \\
\hline$<25.0$ & $11,298(95.3)$ & $557(4.7)$ & & \\
\hline Moderate/severe physical activity $(\%)^{\dagger}$ & & & 162.861 & $<0.001$ \\
\hline Yes & $14,859(91.1)$ & $1457(8.9)$ & & \\
\hline No & $21,719(87.0)$ & $3249(13.0)$ & & \\
\hline Hypertension $(\%)^{+}$ & & & 537.915 & $<0.001$ \\
\hline Yes & $14,672(84.7)$ & $2648(15.3)$ & & \\
\hline No & $19,138(92.2)$ & $1611(7.8)$ & & \\
\hline The doctor told you that you had diabetes (\%) & & & $18,424.978$ & $<0.001$ \\
\hline Yes & $1711(33.1)$ & $3462(66.9)$ & & \\
\hline Borderline & $676(76.2)$ & $211(23.8)$ & & \\
\hline No & $34,207(97.1)$ & $1035(2.9)$ & & \\
\hline Had at least 12 cups of alcoholic drink per year $(\%) f^{\dagger}$ & & & 157.612 & $<0.001$ \\
\hline Yes & $25,714(89.8)$ & $2932(10.2)$ & & \\
\hline No & $8981(85.2)$ & $1559(14.8)$ & & \\
\hline Consumed over 100 cigarettes in lifetime $(\%)^{+}$ & & & 26.543 & $<0.001$ \\
\hline Yes & $16,589(87.7)$ & $2321(12.3)$ & & \\
\hline No & $19,979(89.3)$ & $2383(10.7)$ & & \\
\hline Food security (\%) & & & 38.584 & $<0.001$ \\
\hline Yes & $26,893(89.4)$ & $3174(10.6)$ & & \\
\hline No & $5300(86.7)$ & $812(13.3)$ & & \\
\hline $\mathrm{PIR}^{*}+$ & $2.2(1.2-4.2)$ & $1.8(1.0-3.3)$ & 6.236 & $<0.001$ \\
\hline Energy (kcal) & $1948.0(1441.1-2612.0)$ & $1725.0(1257.0-2329.0)$ & 7.599 & $<0.001$ \\
\hline Protein (gm) & $72.3(51.8-100.7)$ & $69.0(49.3-94.0)$ & 3.809 & $<0.001$ \\
\hline Carbohydrate (gm) & $236.6(170.6-319.7)$ & $204.5(147.4-278.1)$ & 8.295 & $<0.001$ \\
\hline Total fat (gm) & $71.3(47.6-102.3)$ & $64.6(42.7-96.2)$ & 4.666 & $<0.001$ \\
\hline Dietary fiber (gm) & $14.3(9.3-21.2)$ & $14.1(9.3-20.8)$ & 1.140 & 0.148 \\
\hline Thiamin (Vitamin B1) (mg) & $1.4(1.0-2.0)$ & $1.4(0.9-1.9)$ & 2.685 & $<0.001$ \\
\hline Riboflavin (Vitamin B2) (mg) & $1.8(1.3-2.6)$ & $1.7(1.2-2.3)$ & 4.151 & $<0.001$ \\
\hline Vitamin B6 (mg) & $1.7(1.1-2.5)$ & $1.6(1.1-2.3)$ & 4.416 & $<0.001$ \\
\hline Total folate (mcg) & $341.0(230.0-496.0)$ & $320.0(215.0-459.0)$ & 3.558 & $<0.001$ \\
\hline Vitamin B12 (mcg) & $3.7(2.1-6.2)$ & $3.4(1.9-5.6)$ & 3.452 & $<0.001$ \\
\hline Vitamin E (mg) & $6.5(4.2-10.0)$ & $6.1(3.8-9.3)$ & 3.294 & $<0.001$ \\
\hline Calcium (mg) & $779.0(496.0-1151.0)$ & $713.0(468.0-1040.8)$ & 4.185 & $<0.001$ \\
\hline Magnesium (mg) & $265.0(190.0-363.0)$ & $249.0(180.0-336.0)$ & 4.041 & $<0.001$ \\
\hline Iron (mg) & $12.8(8.9-18.4)$ & $12.3(8.5-17.5)$ & 2.466 & $<0.001$ \\
\hline Zinc (mg) & $9.7(6.6-14.1)$ & $8.9(6.1-13.0)$ & 3.824 & $<0.001$ \\
\hline Copper (mg) & $1.1(0.8-1.5)$ & $1.0(0.7-1.4)$ & 4.124 & $<0.001$ \\
\hline Insulin $(\mathrm{uU} / \mathrm{mL})^{+}$ & $9.5(6.2-15.2)$ & $14.7(8.8-25.0)$ & 10.705 & $<0.001$ \\
\hline Glucose $(\mathrm{mg} / \mathrm{dL})^{\dagger}$ & $91.0(85.0-99.0)$ & $149.0(118.0-149.0)$ & 45.353 & $<0.001$ \\
\hline Hemoglobin $(\mathrm{g} / \mathrm{dL})^{\dagger}$ & $14.2(13.2-15.2)$ & $13.9(12.8-15.0)$ & 5.840 & $<0.001$ \\
\hline
\end{tabular}

\# The statistical description of the two groups was expressed in the form of a number (\%)/median ( $25 \%$ percentile- $75 \%$ percentile). ${ }^{\dagger}$ There were missing values in the two groups. $\ddagger$ One cup of alcoholic drink is equivalent to 12 ounces of beer, 4 ounces of wine, or an ounce of liquor. * BMI, body mass index; PIR, poverty income ratio; RGCS, recent glycemic control states. 

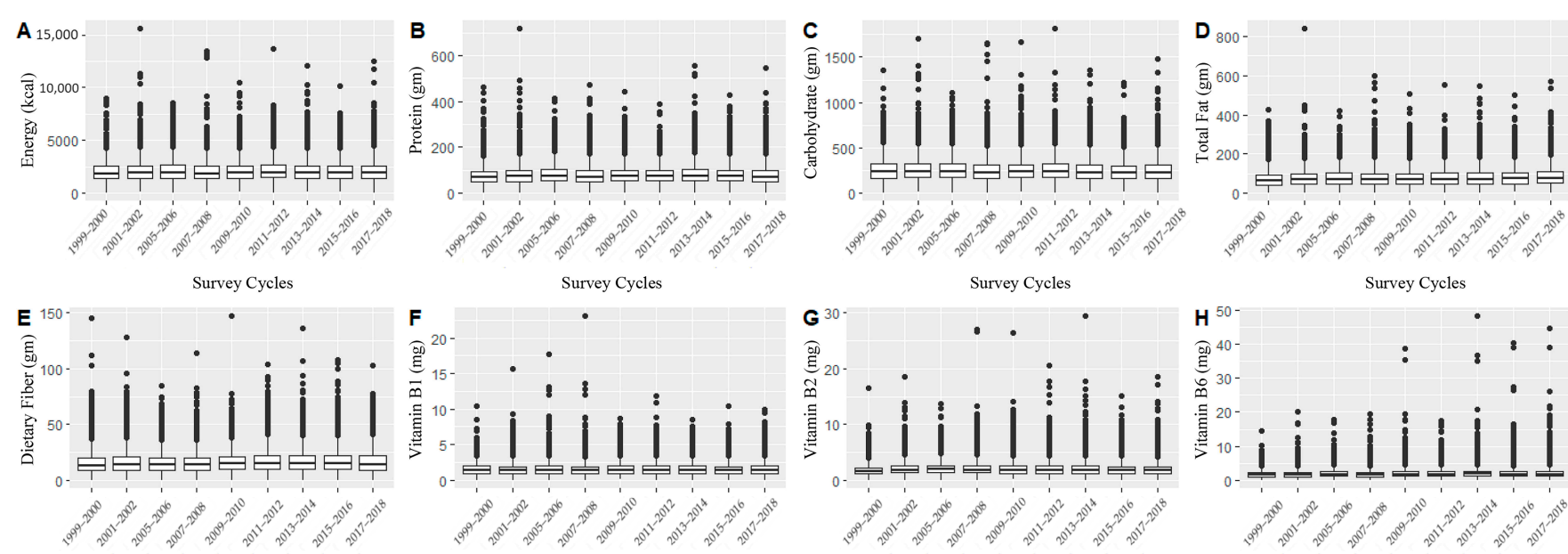

Survey Cycles
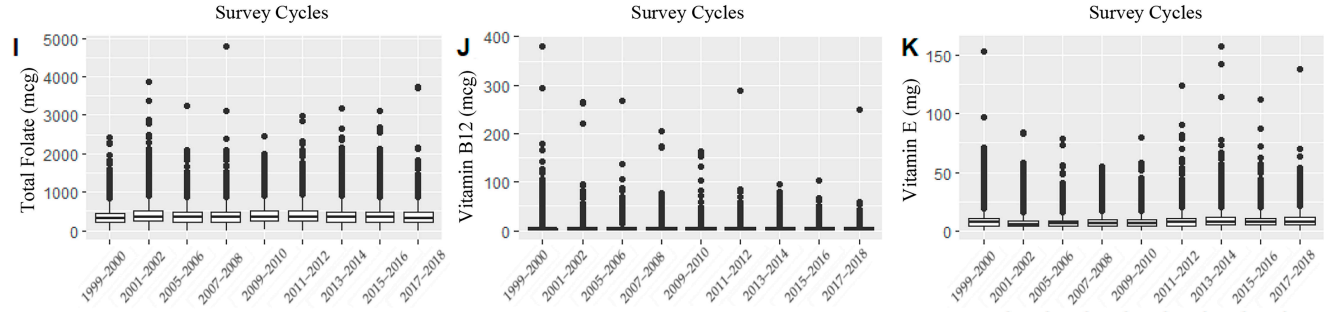

Survey Cycles

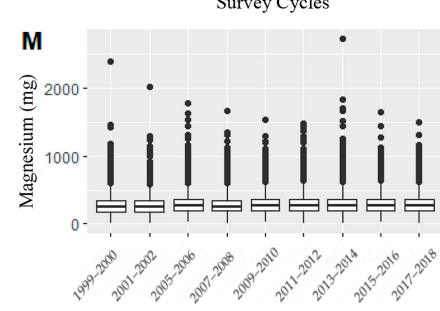

Survey Cycles

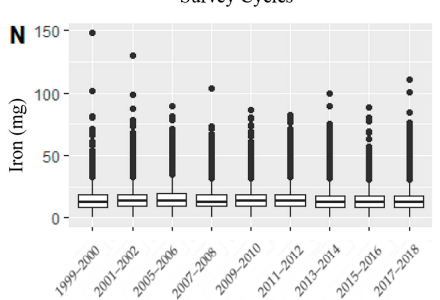

Survey Cycles
Survey Cycles

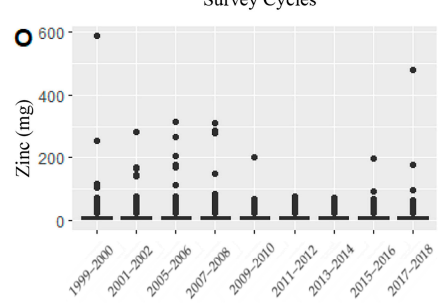

Survey Cycles

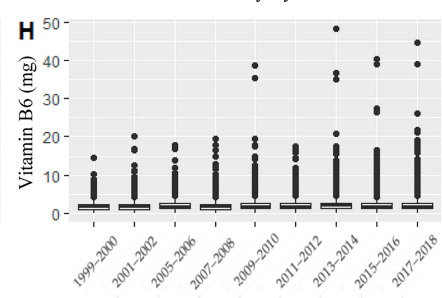

Survey Cycles

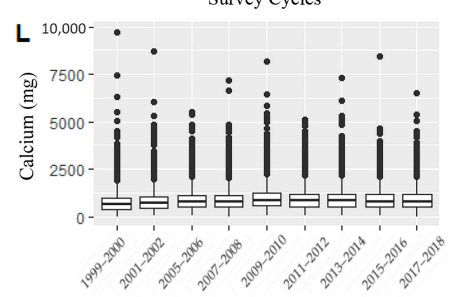

Survey Cycles

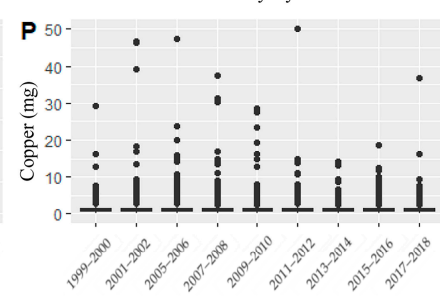

Survey Cycles

Figure 1. Distribution of daily total dietary energy and nutrient intakes in different investigation periods ((A) Energy (kcal); (B) Protein (gm); (C) Carbohydrate; (D) Total Fat (gm); (E) Dietary Fiber (gm); (F) Vitamin B1 (mg); (G) Vitamin B2 (mg); (H) Vitamin B6 (mg); (I) Total Folate (mg); (J) Vitamin B12 (mg); (K) Vitamin E (mg); (L) Calcium (mg); (M) Magnesium (mg); (N) Iron (mg); (O) Zinc (mg); (P) Copper (mg)).

\subsection{Binary Logistic Regression Analysis}

3.2.1. The Association between RGCS and Daily Total Dietary Energy, Macronutrients, Vitamins, and Minerals

For the crude model and adjusted model I, we found that energy, protein, total fat, dietary fiber, vitamin B1, vitamin B6, vitamin E, iron, and zinc intake were associated with RGCS, including good RGCS and poor RGCS (Figure 2A,B). However, after controlling of all covariates and time fixed effect (Figure 2C,D), opposite results were obtained from the former two models, for instance, three kinds of macronutrients, minerals, and dietary fiber actually had no statistical association with RGCS. Eventually, the statistical results in the robust check model (Figure 2D and Table 2), after controlling for the potential confounders and years fixed effect, suggested a significantly negative correlation between daily total dietary vitamin B6 intake and RGCS (adjusted OR = 0.848; 95\% CI: 0.738, 0.973; $p$-value $=0.019)$. 
A

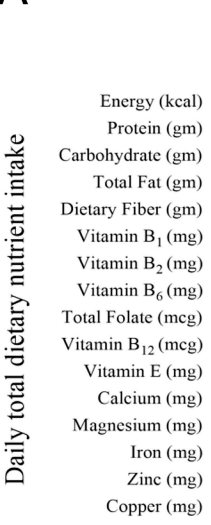

C

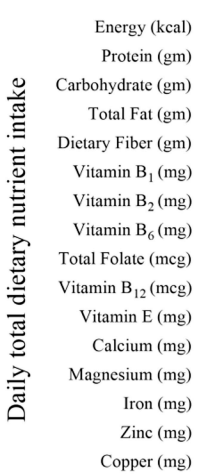

Crude Model

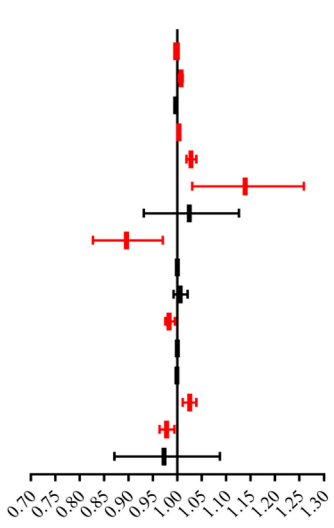

Adjusted Model Two

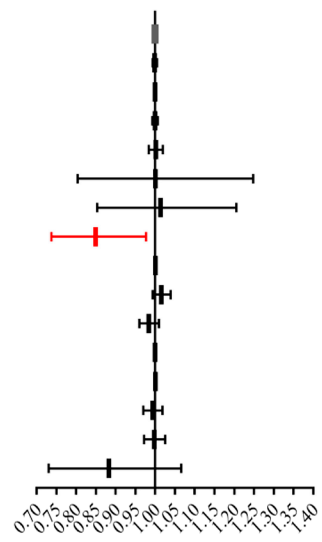

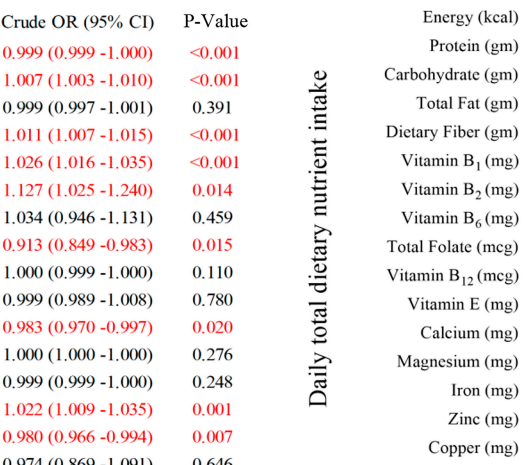

Adjusted OR (95\% CI) P-Value

$1.000(0.999-1.001) \quad 0.910$

$1.001(0.995-1.006) \quad 0.836$

$1.000(0.998-1.003)$

$1.000(0.993-1.006)$

$1.004(0.986-1.021)$

$0.990(0.802-1.221)$

$1.017(0.861-1.202)$

$0.855(0.746-0.981)$

$1.001(1.000-1.001)$

$1.006(0.990-1.021)$

$0.987(0.963-1.011)$

$1.000(1.000-1.000)$

$1.001(0.999-1.002)$

$0.998(0.975-1.022)$

$0.993(0.968-1.019)$

$0.926(0.782-1.097)$
B

D

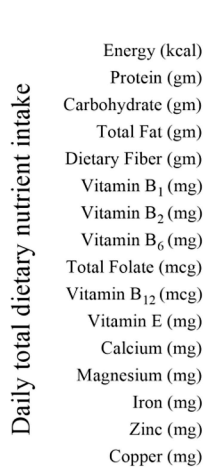

Adjusted Model One

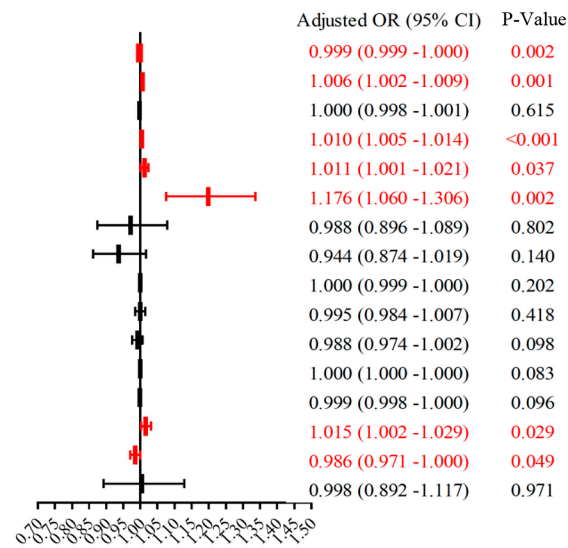

Robust Check Model

Robust OR (95\% CI) P-Value

$1.000(0.999-1.001) \quad 0.828$ $1.001(0.995-1.006) \quad 0.845$ $1.000(0.998-1.003) \quad 0.823$ $1.000(0.993-1.007) \quad 0.969$ $1.003(0.986-1.021)-0.730$ $0.989(0.800-1.223)-0.919$ $1.026(0.868-1.213) \quad 0.762$ $\begin{array}{ll}0.848(0.738-0.973) & 0.019\end{array}$ $1.001(1.000-1.001) \quad 0.122$ $1.005(0.989-1.021) \quad 0.520$ $0.986(0.961-1.011) \quad 0.261$ $1.000(1.000-1.000)-0.877$ $1.001(0.999-1.002) \quad 0.458$ $1.000(0.977-1.024) \quad 0.974$ $0.995(0.970-1.020) \quad 0.680$ $0.941(0.789-1.122) \quad 0.497$

Figure 2. Forest plot for odds ratio (OR) and 95\% confidence interval (CI) of daily total dietary nutrient and energy intake ((A) without covariates; (B) gender, age, and race were controlled; (C) all potential confounders in the study were controlled; (D) all potential confounders and the years fixed effect in the study were controlled).

Table 2. Binary logistic regression analysis between RGCS and dietary nutrient and energy intake among non-pregnant adults 20+ years old from NHANES 1999-2018 (except for 2003-2004).

\begin{tabular}{ccccc}
\hline Variables & Crude Model $^{\mathbf{a}}$ & Model I $^{\mathbf{b}}$ & Model II $^{\mathbf{c}}$ & Robust Check Model $^{\mathbf{d}}$ \\
\cline { 2 - 5 } & $\boldsymbol{\beta}(\mathrm{SE})$ & $\beta(S E)$ & $\beta(S E)$ & $\beta(S E)$ \\
\hline Energy (kcal) & $-0.001(0.0002)^{* * *}$ & $-0.001(0.0002)^{* * *}$ & $-0.00003(0.0003)$ & $-0.00006(0.0003)$ \\
Protein (gm) & $0.007(0.002)^{* * *}$ & $0.006(0.002)^{* *}$ & $0.001(0.003)$ & $0.001(0.003)$ \\
Carbohydrate (gm) & $-0.001(0.001)$ & $-0.0005(0.001)$ & $0.0001(0.001)$ & $0.0003(0.001)$ \\
Total fat (gm) & $0.011(0.002)^{* * *}$ & $0.010(0.002)^{* * *}$ & $-0.0005(0.003)$ & $-0.0001(0.003)$ \\
Dietary fiber (gm) & $0.025(0.005)^{* * *}$ & $0.011(0.005)^{*}$ & $0.004(0.009)$ & $0.003(0.009)$ \\
Thiamin (Vitamin B1) (mg) & $0.120(0.049)^{*}$ & $0.162(0.053)^{* *}$ & $-0.011(0.107)$ & $-0.011(0.108)$ \\
Riboflavin (Vitamin B2) (mg) & $0.034(0.045)$ & $-0.012(0.050)$ & $0.017(0.085)$ & $0.026(0.085)$ \\
Vitamin B6 (mg) & $-0.091(0.037)^{*}$ & $-0.058(0.039)$ & $-0.157(0.070)^{*}$ & $-0.165(0.070)^{*}$ \\
Total folate (mcg) & $-0.0004(0.0002)$ & $-0.0003(0.0002)$ & $0.001(0.0004)$ & $0.001(0.0004)$ \\
Vitamin B12 (mcg) & $-0.001(0.005)$ & $-0.005(0.006)$ & $0.006(0.008)$ & $0.005(0.008)$ \\
Vitamin E (mg) & $-0.017(0.007)^{*}$ & $-0.012(0.007)$ & $-0.013(0.013)$ & $-0.014(0.013)$ \\
Calcium (mg) & $-0.00008(0.00008)$ & $0.0001(0.00008)$ & $0.00002(0.0001)$ & $-0.00002(0.0001)$ \\
Magnesium (mg) & $-0.001(0.0005)$ & $-0.001(0.0005)$ & $0.001(0.001)$ & $0.001(0.001)$ \\
Iron (mg) & $0.022(0.006)^{* *}$ & $0.015(0.007)^{*}$ & $-0.002(0.012)$ & $0.0004(0.012)$ \\
Zinc (mg) & $-0.020(0.007)^{* *}$ & $-0.015(0.007)^{*}$ & $-0.007(0.013)$ & $-0.005(0.013)$ \\
Copper (mg) & $-0.027(0.058)$ & $-0.002(0.057)$ & $-0.077(0.086)^{* *}$ & $-0.061(0.090)$ \\
\hline
\end{tabular}


Table 2. Cont.

\begin{tabular}{|c|c|c|c|c|}
\hline \multirow{2}{*}{ Variables } & Crude Model ${ }^{a}$ & Model I ${ }^{b}$ & Model II ${ }^{c}$ & Robust Check Model ${ }^{d}$ \\
\hline & $\beta(S E)$ & $\beta(S E)$ & $\beta(S E)$ & $\beta(S E)$ \\
\hline Age $(<40$ years old $)$ & - & Reference & Reference & Reference \\
\hline Age $(40-59$ years old $)$ & - & $2.211(0.103)^{* * *}$ & $0.985(0.190)^{* * *}$ & $0.978(0.191) * * *$ \\
\hline Age ( $\geq 60$ years old $)$ & - & $1.561(0.104)^{* * *}$ & $0.792(0.189) * * *$ & $0.782(0.189) * * *$ \\
\hline Gender (male) & - & $0.230(0.058) * * *$ & $0.322(0.117)^{* *}$ & $0.311(0.118) * *$ \\
\hline Race (other races) & - & Reference & Reference & Reference \\
\hline Race (Mexican American) & - & $-0.551(0.083)^{* * *}$ & $-0.269(0.150)$ & $-0.229(0.151)$ \\
\hline Race (non-Hispanic Black) & - & $0.298(0.089)^{* *}$ & $0.342(0.163)$ * & $0.396(0.164) *$ \\
\hline Race (non-Hispanic White) & - & $0.209(0.091) *$ & $-0.024(0.170)$ & $0.043(0.172)$ \\
\hline Education level ( $\leq$ high school) & - & - & $0.179(0.107)$ & $0.187(0.108)$ \\
\hline $\mathrm{BMI}^{+}(<25.0)$ & - & - & Reference & Reference \\
\hline BMI (25.0-29.9) & - & - & $0.726(0.146) * * *$ & $0.714(0.146)^{* * *}$ \\
\hline $\mathrm{BMI}(\geq 30.0)$ & - & - & $0.081(0.156)$ & $0.076(0.156)$ \\
\hline $\begin{array}{c}\text { Moderate/severe physical } \\
\text { activity (no) }\end{array}$ & - & - & $0.114(0.103)$ & $0.076(0.104)$ \\
\hline Hypertension (yes) & - & - & $0.296(0.100) * *$ & $0.323(0.100)$ \\
\hline $\begin{array}{l}\text { The doctor told you that you had } \\
\text { diabetes (no) }\end{array}$ & - & - & Reference & Reference \\
\hline $\begin{array}{l}\text { The doctor told you that you had } \\
\text { diabetes (borderline) }\end{array}$ & - & - & $2.501(0.106)^{* * *}$ & $2.491(0.106)^{* * *}$ \\
\hline $\begin{array}{l}\text { The doctor told you that you had } \\
\text { diabetes (yes) }\end{array}$ & - & - & $1.167(0.196)^{* * *}$ & $1.141(0.196)^{* * *}$ \\
\hline $\begin{array}{l}\text { Had at least } 12 \text { cups alcoholic } \\
\text { drink per year (yes) }\end{array}$ & - & - & $-0.057(0.112)$ & $-0.077(0.113)$ \\
\hline $\begin{array}{c}\text { Consumed over } 100 \text { cigarettes in } \\
\text { their lifetime (yes) }\end{array}$ & - & - & $-0.085(0.103)$ & $-0.073(0.104)$ \\
\hline Food security (no) & - & - & $0.076(0.135)$ & $0.047(0.137)$ \\
\hline $\mathrm{PIR}^{+}$ & - & - & $-0.049(0.036)$ & $-0.048(0.036)$ \\
\hline Insulin (uU/mL) & - & - & $0.003(0.002)$ & $0.003(0.002)$ \\
\hline Glucose (mg/dL) & - & - & $0.061(0.002)^{* * *}$ & $0.061(0.002)^{* * *}$ \\
\hline Hemoglobin (g/dL) & - & - & $-0.095(0.036) * *$ & $-0.086(0.036) *$ \\
\hline Years fixed effect & - & - & - & Included \\
\hline
\end{tabular}

a A total of 15 dietary variables were entered in the crude model: protein, carbohydrate, total fat, dietary fiber, vitamin b1, vitamin b2, vitamin b6, total folate, vitamin b12, vitamin e, calcium, magnesium, iron, zinc, copper. ${ }^{\mathrm{b}}$ Three variables were adjusted in model I: gender, age, race. ${ }^{c}$ A total of 17 variables were adjusted in model II: gender, age, race, education level, BMI, moderate or severe physical activity, hypertension, the doctor informing them that they had diabetes, having at least 12 cups of alcoholic drink per year, consuming over 100 cigarettes in their lifetime, food security, PIR, energy, insulin, glucose, hemoglobin. ${ }^{\mathrm{d}}$ Robust check model: Based on model II, years fixed effect was adjusted. ${ }^{*} p$-value $<0.05 ;{ }^{* *} p$-value $<0.01 ;{ }^{* * *} p$-value $<0.001 .{ }^{\dagger}$ BMI, body mass index; PIR, poverty income ratio; RGCS, recent glycemic control states; $\mathrm{SE}$, standard error.

\subsubsection{The Association between Adjusted Covariates and RGCS}

The statistical results of the robust check model, all covariates, and time fixed effect being adjusted demonstrated that age (taking " $<40$ years old" as a reference, $\mathrm{OR}_{[40-59 \text { years old] }}=2.659$, $p$-value $<0.001 ; \mathrm{OR}_{[\geq 60 \text { years old }]}=2.186, p$-value $<0.001$ ), gender (taking "female" as a reference, $\mathrm{OR}_{[\text {Male }]}=1.365, p$-value $=0.008$ ), race (taking "other races" as a reference, $\mathrm{OR}_{[\text {Mexican American }]}=0.796, p$-value $=0.131 ; \mathrm{OR}_{[\text {Non-Hispanic Black }]}=1.486, p$-value $=0.016$; $\mathrm{OR}_{[\text {Non-Hispanic White] }}=1.044, p$-value $=0.804$ ), education level (taking "> high school" as a reference, $\mathrm{OR}_{[\leq \text {High School] }}=1.205, p$-value $=0.083$ ), BMI (taking " $<25.0$ " as a reference, $\mathrm{OR}_{[25.0-29.9]}=2.042, p$-value $<0.001 ; \mathrm{OR}_{[\geq 30.0]}=1.079, p$-value $\left.=0.626\right)$, hypertension (taking "no" as a reference, $\mathrm{OR}_{[\text {Yes] }}=1.381, p$-value $=0.001$ ), the doctor informing them that they had diabetes (taking "no" as a reference, $\mathrm{OR}_{\text {[Borderline] }}=12.072, p$-value $<0.001$; $\mathrm{OR}_{[\text {Yes] }}=3.130, p$-value $\left.<0.001\right)$, insulin $(\mathrm{OR}=1.003, p$-value $=0.132)$, glucose $(\mathrm{OR}=1.063$, $p$-value $<0.001)$, and hemoglobin $(\mathrm{OR}=0.918, p$-value $=0.017)$ were significantly associated with RGCS in Table 2. 


\subsection{Model Fitting, Linear Discriminant Analysis, and ROC Analysis}

3.3.1. Model Fitting and Linear Discriminant Analysis of Daily Total Dietary Vitamin B6 Intake, Glycohemoglobin, and RGCS

After smooth curve fitting of daily total dietary vitamin B6 intake and glycohemoglobin being conducted in Figure 3, the robust check model, a linear discriminant analysis of daily total dietary vitamin B6 intake and RGCS, was also fitted in Figure 4. The statistical analysis graphs showed that the established robust check model could not only distinguish American adults with different RGCS well, but pointed out that the negative correlation between daily total dietary vitamin B6 intake and RGCS did exist. It was indicated that daily total dietary vitamin B6 intake might have a potential predictive value for RGCS of American adults.
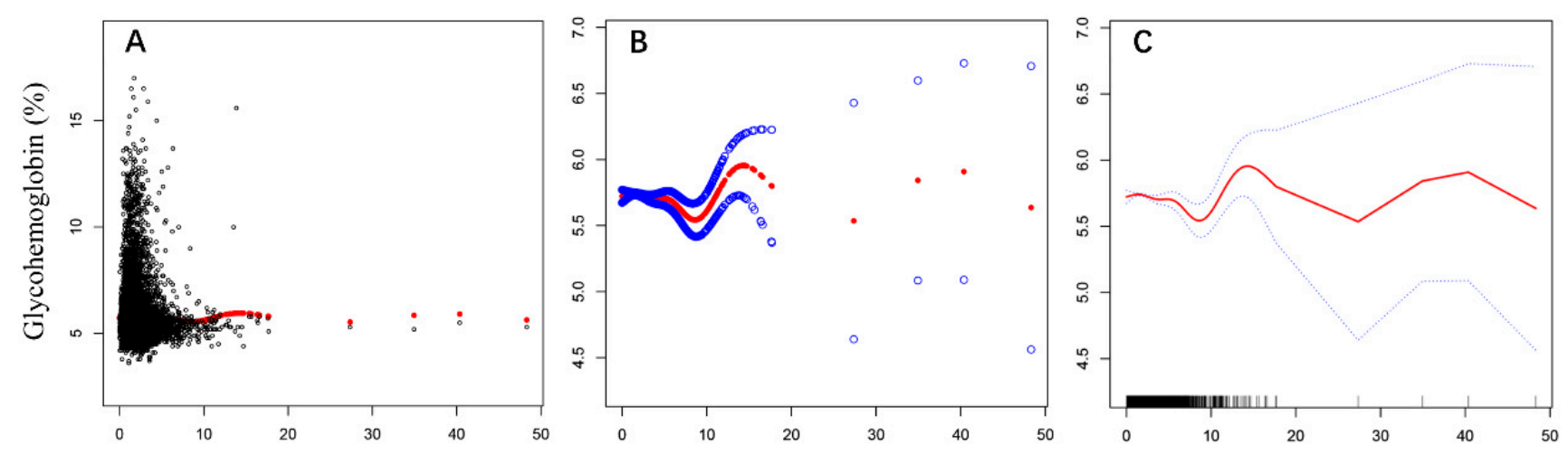

Total dietary vitamin $\mathrm{B} 6$ intake $(\mathrm{mg} / \mathrm{d})$

Figure 3. The model fitting processes of non-linear regression curve ((A,B), scatter plots; $(\mathbf{C})$, the optimal smooth curve).
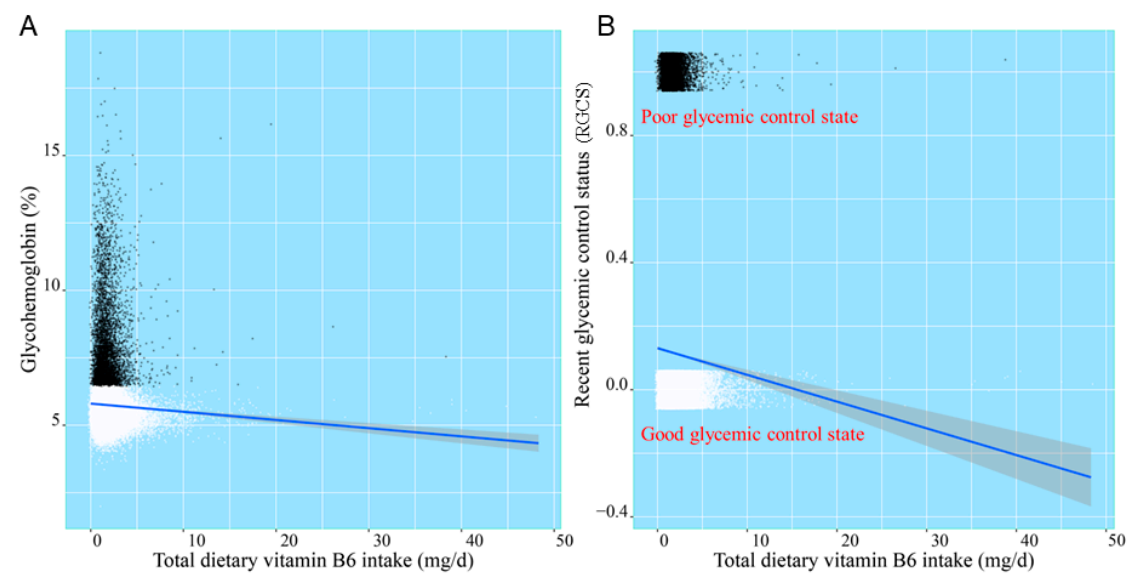

Figure 4. The linear discriminant analysis of daily total dietary vitamin B6 intake, glycohemoglobin (A), and RGCS (B).

\subsubsection{ROC Analysis of Daily Total Dietary Vitamin B6 Intake}

ROC analysis of daily total dietary vitamin B6 intake was performed to calculate the area under the curve (AUC), which was used to evaluate the discrimination accuracy among people with good and poor RGCS. As shown in Figure 5, after controlling for all potential confounders and the years fixed effect, the predictive potential or accuracy of the multivariate logistic regression robust check model (AUC $=0.977 ; 95 \%$ CI: 0.974, 0.980; $p$-value $<0.001$ ) was higher than those of the crude model (AUC $=0.535 ; 95 \%$ CI: 0.519 , $0.550 ; p$-value < 0.001), adjusted model I (AUC $=0.710 ; 95 \%$ CI: 0.697, 0.723; $p$-value < 0.001 ), and adjusted model II (AUC $=0.975 ; 95 \%$ CI: 0.974, $0.979 ; p$-value $<0.001$ ). The test results of DeLong between robust check model and crude model, and adjusted model I and adjusted model II showed that there were two statistically significant results (robust check 
model vs. crude model, $Z=53.54, p$-value $<0.001$; robust check model vs. adjusted model I, $Z=39.542, p$-value $<0.001$; robust check model vs. adjusted model II, $Z=0.751$, $p$-value $=0.452)$.
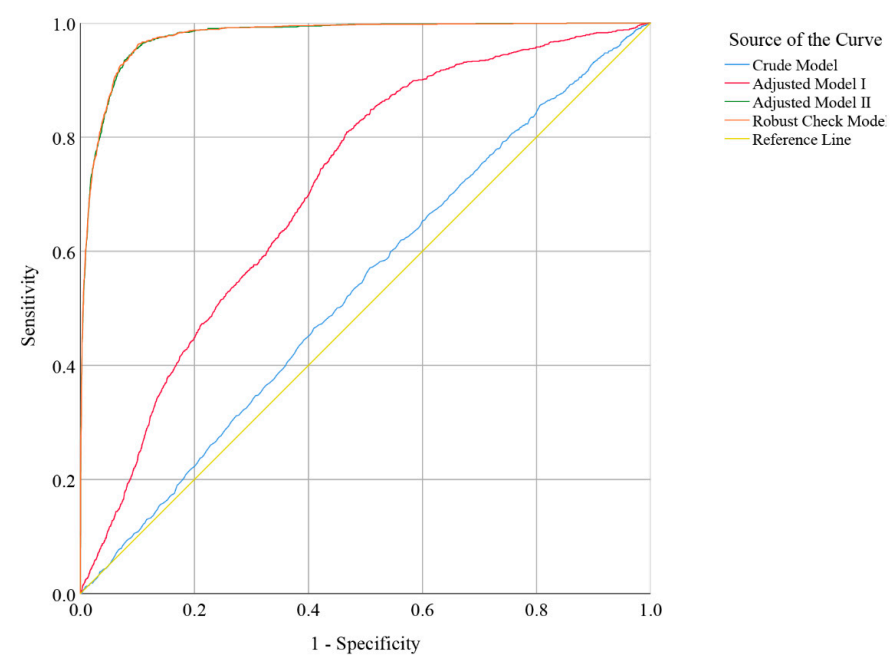

Figure 5. Receiver operator characteristic (ROC) curve indicator of poor RGCS (crude model: without covariates; adjusted model I: gender, age, and race were controlled; adjusted model II: all potential confounders in the study were controlled; robust check model: all potential confounders in the study were controlled, and the years fixed effect in the study was included).

\section{Discussion}

Although the reliable data of our study were from the national representative sample published by the Centers for Disease Control and Prevention of the United States, there were still some missing values in our collected data, which might have a subtle influence on our results of the statistical analysis. However, given the sufficient sample size of this study, the deviation caused by missing values could be reduced. Therefore, the reliability and authenticity of our findings were within acceptable limits. In addition, we did not ignore the interactions among nutrients when fitting the saturation model, but these interactions that might have biological significance (such as the interaction between vitamin B6 and vitamin B12) were not statistically significant when they were included in the robust check model.

The statistical model constructed in our research combined the specific macronutrients, minerals, vitamins, dietary fiber, and energy of the daily total diet, demographic, and medical indicators of American adults, because some statistical models mentioned in other studies might only be relevant for a small group of people with diabetes and not be suitable for American adults in terms of predicting their RGCS. Thus, our robust check model was closer to the real-world results than the models established by those research institutes $[27,29]$.

Eventually, we found only daily total dietary vitamin B6 intake negatively correlated with RGCS, that is, the higher the daily total intake of dietary vitamin B6 was accompanied with better RGCS. Similarly, Mascolo et al. also concluded that the vitamin B6 level was significantly negatively associated with diabetes mellitus in diabetic people and suggested that vitamin B6 had a significantly protective effect on diabetic complications [30]. In addition, although covariates adjusted in our robust check model could not answer our research issues, they could still provide the theoretical foundation and scientific guidance for our health education related to glycemic control for American adults, which had a valuable public health significance.

The establishment of the robust check model of nutrients and RGCS was only the preliminary step of this study, which was mainly used to qualitatively find the associated factors affecting the RGCS. After screening the statistically significant daily total dietary 
vitamin B6 intake with this model, we further performed linear discriminant model and ROC analysis between RGCS and daily total dietary vitamin B6 intake as well as HbA1c, respectively (Figures 4 and 5), which could provide a quantitative reference and prediction accuracy of daily total dietary vitamin B6 intake for American adults who need to control blood glucose.

Vitamin B6 was an intriguing molecule that was involved in a wide range of metabolic, physiological, and developmental processes. Its active form, 5'-pyridoxal phosphate (PLP), was a co-factor for approximately 150 metabolic responses to glucose, lipid, amino acids, DNA, and neurotransmitters [30-34]. These studies showed that vitamin B6 had a potential to regulate body metabolism (including blood glucose). Although the United States, South Korea, and Japan published the recommended total dietary intake of vitamin B6 (fluctuating around 1.1 1.6 mg/d) for specific populations [35-37], it might not be suitable for American adults who need to control blood glucose. Therefore, it was necessary for the relevant health management agencies in the United States to formulate the recommended value of the total daily dietary vitamin B6 intake of RGC for American adults, so as to provide an effective way for them to obtain better RGCS. However, the formulation of total daily dietary vitamin $\mathrm{B} 6$ recommended intake remains to be further explored.

\section{Conclusions}

In summary, our results indicated that only daily total dietary vitamin B6 intake was significant negatively associated with RGCS among all dietary nutrients we studied. Although this study provided a ROC prediction result of daily total dietary vitamin B6 intake for RGCS, we might require further validation of whether it would have a positive and effective preventive effect and biological implications on RGCS of American adults.

Supplementary Materials: The following are available online at https:/ / www.mdpi.com/article/1 0.3390/nu13114168/s1, Figure S1: Participant flow chart.

Author Contributions: The authors' responsibilities were as follows-Y.B., L.P.: contributed to data collection, analysis, and manuscript writing; H.Z., J.Y.: participated in the research design; L.P.: devoted to research design and manuscript writing. All authors have read and agreed to the published version of the manuscript.

Funding: The research received no sources of support such as commercial or non-commercial funding. The findings and conclusions expressed in this article were the authors' and do not necessarily represent the official position of the CDC or the U.S. Department of Health and Human Services. There was no private sponsor who played any role in the decision to design the study, collect data, analyze or interpret data, write reports, or submit manuscripts.

Institutional Review Board Statement: Ethical review and approval were waived for this study, since all the data from National Health and Nutrition Examination Survey is publicly accessible.

Informed Consent Statement: Informed consent from all subjects was obtained by National Health and Nutrition Examination Survey.

Data Availability Statement: Data described in the manuscript, codebook, and analytic code will not be made available because the data used in this study were from the NHANES database, which is a free and open database for all researchers around the world.

Conflicts of Interest: The authors stated that no conflict of interest would be deemed to prejudice the impartiality of the reported study.

\section{Abbreviations}

AUC: area under the curve; BMI, body mass index; $\mathrm{CI}$, confidence interval; $\mathrm{HbA1c}$, glycosylated hemoglobin; NCHS, National Center for Health Statistics; NHANES, National Health and Nutrition Examination Survey; OR, odds ratio; PIR, poverty income ratio; RGCS, recent glycemic control status; ROC, receiver operator characteristic; SE, standard error; USDA, United States Department of Agriculture; VIF, variance inflation factor. 


\section{References}

1. Venn, B.J. Macronutrients and Human Health for the 21st Century. Nutrients 2020, 12, 2363. [CrossRef]

2. Franz, M.J. Diabetes Nutrition Therapy: Effectiveness, Macronutrients, Eating Patterns and Weight Management. Am. J. Med Sci. 2016, 351, 374-379. [CrossRef] [PubMed]

3. Scott, S.; Kempf, P.; Bally, L.; Stettler, C. Carbohydrate Intake in the Context of Exercise in People with Type 1 Diabetes. Nutrients 2019, 11, 3017. [CrossRef] [PubMed]

4. Fuller, S.; Beck, E.; Salman, H.; Tapsell, L. New Horizons for the Study of Dietary Fiber and Health: A Review. Plant Foods Hum. Nutr. 2016, 71, 1-12. [CrossRef] [PubMed]

5. Meyer, K.A.; Kushi, L.H.; Jacobs, D.R., Jr.; Slavin, J.; Sellers, T.A.; Folsom, A.R. Carbohydrates, dietary fiber, and incident type 2 diabetes in older women. Am. J. Clin. Nutr. 2000, 71, 921-930. [CrossRef]

6. Yu, K.; Ke, M.Y.; Li, W.H.; Zhang, S.Q.; Fang, X.C. The impact of soluble dietary fibre on gastric emptying, postprandial blood glucose and insulin in patients with type 2 diabetes. Asia Pac. J. Clin. Nutr. 2014, 23, 210-218. [CrossRef]

7. Lin, C.C.; Huang, Y.L. Chromium, zinc and magnesium status in type 1 diabetes. Curr. Opin. Clin. Nutr. Metab. Care 2015, 18, 588-592. [CrossRef]

8. Skalnaya, M.G.; Skalny, A.V.; Grabeklis, A.R.; Serebryansky, E.P.; Demidov, V.A.; Tinkov, A.A. Hair Trace Elements in Overweight and Obese Adults in Association with Metabolic Parameters. Biol. Trace Elem. Res. 2018, 186, 12-20. [CrossRef]

9. Soliman, A.T.; De Sanctis, V.; Yassin, M.; Soliman, N. Iron deficiency anemia and glucose metabolism. Acta Bio-Med. Atenei Parm. 2017, 88, 112-118. [CrossRef]

10. Deshmukh, S.V.; Prabhakar, B.; Kulkarni, Y.A. Water Soluble Vitamins and their Role in Diabetes and its Complications. Curr. Diabetes Rev. 2020, 16, 649-656. [CrossRef] [PubMed]

11. Kaur, B.; Henry, J. Micronutrient status in type 2 diabetes: A review. Adv. Food Nutr. Res. 2014, 71, 55-100. [CrossRef]

12. Li, X.; Liu, Y.; Zheng, Y.; Wang, P.; Zhang, Y. The Effect of Vitamin D Supplementation on Glycemic Control in Type 2 Diabetes Patients: A Systematic Review and Meta-Analysis. Nutrients 2018, 10, 375. [CrossRef] [PubMed]

13. Lips, P.; Eekhoff, M.; van Schoor, N.; Oosterwerff, M.; de Jongh, R.; Krul-Poel, Y.; Simsek, S. Vitamin D and type 2 diabetes. J. Steroid Biochem. Mol. Biol. 2017, 173, 280-285. [CrossRef]

14. Fazelian, S.; Rouhani, M.H.; Bank, S.S.; Amani, R. Chromium supplementation and polycystic ovary syndrome: A systematic review and meta-analysis. J. Trace Elem. Med. Biol. Organ Soc. Miner. Trace Elem. (GMS) 2017, 42, 92-96. [CrossRef] [PubMed]

15. Suksomboon, N.; Poolsup, N.; Yuwanakorn, A. Systematic review and meta-analysis of the efficacy and safety of chromium supplementation in diabetes. J. Clin. Pharm. Ther. 2014, 39, 292-306. [CrossRef]

16. Yin, R.V.; Phung, O.J. Effect of chromium supplementation on glycated hemoglobin and fasting plasma glucose in patients with diabetes mellitus. Nutr. J. 2015, 14, 14. [CrossRef]

17. Li, Z.; Xu, Y.; Huang, Z.; Wei, Y.; Hou, J.; Long, T.; Wang, F.; Hu, H.; Duan, Y.; Guo, H.; et al. Association between exposure to arsenic, nickel, cadmium, selenium, and zinc and fasting blood glucose levels. Environ. Pollut. 2019, 255, 113325. [CrossRef]

18. Wang, X.; Wu, W.; Zheng, W.; Fang, X.; Chen, L.; Rink, L.; Min, J.; Wang, F. Zinc supplementation improves glycemic control for diabetes prevention and management: A systematic review and meta-analysis of randomized controlled trials. Am. J. Clin. Nutr. 2019, 110, 76-90. [CrossRef] [PubMed]

19. Simental-Mendía, L.E.; Sahebkar, A.; Rodríguez-Morán, M.; Guerrero-Romero, F. A systematic review and meta-analysis of randomized controlled trials on the effects of magnesium supplementation on insulin sensitivity and glucose control. Pharmacol. Res. 2016, 111, 272-282. [CrossRef]

20. Volpe, S.L. Magnesium in disease prevention and overall health. Adv. Nutr. 2013, 4, 378s-383s. [CrossRef]

21. Yazdanpanah, S.; Rabiee, M.; Tahriri, M.; Abdolrahim, M.; Rajab, A.; Jazayeri, H.E.; Tayebi, L. Evaluation of glycated albumin (GA) and GA/HbA1c ratio for diagnosis of diabetes and glycemic control: A comprehensive review. Crit. Rev. Clin. Lab. Sci. 2017, 54, 219-232. [CrossRef] [PubMed]

22. Weykamp, C. HbA1c: A review of analytical and clinical aspects. Ann. Lab. Med. 2013, 33, 393-400. [CrossRef]

23. Konishi, K.; Wada, K.; Tamura, T.; Tsuji, M.; Kawachi, T.; Nagata, C. Dietary magnesium intake and the risk of diabetes in the Japanese community: Results from the Takayama study. Eur. J. Nutr. 2017, 56, 767-774. [CrossRef]

24. Bo, S.; Pisu, E. Role of dietary magnesium in cardiovascular disease prevention, insulin sensitivity and diabetes. Curr. Opin. Lipidol. 2008, 19, 50-56. [CrossRef]

25. Gannon, M.C.; Nuttall, F.Q. Effect of a high-protein, low-carbohydrate diet on blood glucose control in people with type 2 diabetes. Diabetes 2004, 53, 2375-2382. [CrossRef]

26. Intra, J.; Limonta, G.; Cappellini, F.; Bertona, M.; Brambilla, P. Glycosylated Hemoglobin in Subjects Affected by Iron-Deficiency Anemia. Diabetes Metab. J. 2019, 43, 539-544. [CrossRef] [PubMed]

27. Song, S.; Lee, J.E. Dietary Patterns Related to Triglyceride and High-Density Lipoprotein Cholesterol and the Incidence of Type 2 Diabetes in Korean Men and Women. Nutrients 2018, 11, 8. [CrossRef]

28. Ahluwalia, N.; Dwyer, J.; Terry, A.; Moshfegh, A.; Johnson, C. Update on NHANES Dietary Data: Focus on Collection, Release, Analytical Considerations, and Uses to Inform Public Policy. Adv. Nutr. 2016, 7, 121-134. [CrossRef] [PubMed]

29. Brandão-Lima, P.N.; Carvalho, G.B.; Santos, R.K.F.; Santos, B.D.C.; Dias-Vasconcelos, N.L.; Rocha, V.S.; Barbosa, K.B.F.; Pires, L.V. Intakes of Zinc, Potassium, Calcium, and Magnesium of Individuals with Type 2 Diabetes Mellitus and the Relationship with Glycemic Control. Nutrients 2018, 10, 1948. [CrossRef] 
30. Mascolo, E.; Vernì, F. Vitamin B6 and Diabetes: Relationship and Molecular Mechanisms. Int. J. Mol. Sci. 2020, 21, 3669. [CrossRef] [PubMed]

31. Bender, D.A. Vitamin B6 requirements and recommendations. Eur. J. Clin. Nutr. 1989, 43, 289-309.

32. Ueland, P.M.; Ulvik, A.; Rios-Avila, L.; Midttun, Ø.; Gregory, J.F. Direct and Functional Biomarkers of Vitamin B6 Status. Annu. Rev. Nutr. 2015, 35, 33-70. [CrossRef] [PubMed]

33. Ueland, P.M.; McCann, A.; Midttun, Ø.; Ulvik, A. Inflammation, vitamin B6 and related pathways. Mol. Asp. Med. 2017, 53, 10-27. [CrossRef]

34. Hellmann, H.; Mooney, S. Vitamin B6: A molecule for human health? Molecules 2010, 15, 442-459. [CrossRef] [PubMed]

35. Horikawa, C.; Aida, R.; Kamada, C.; Fujihara, K.; Tanaka, S.; Tanaka, S.; Araki, A.; Yoshimura, Y.; Moriya, T.; Akanuma, Y.; et al. Vitamin B6 intake and incidence of diabetic retinopathy in Japanese patients with type 2 diabetes: Analysis of data from the Japan Diabetes Complications Study (JDCS). Eur. J. Nutr. 2020, 59, 1585-1594. [CrossRef]

36. Cho, Y.O.; Kim, B.Y. Vitamin B6 intake by Koreans should be based on sufficient amount and a variety of food sources. Nutrition 2005, 21, 1113-1119. [CrossRef] [PubMed]

37. Bolzetta, F.; Veronese, N.; De Rui, M.; Berton, L.; Toffanello, E.D.; Carraro, S.; Miotto, F.; Inelmen, E.M.; Donini, L.M.; Manzato, E.; et al. Are the Recommended Dietary Allowances for Vitamins Appropriate for Elderly People? J. Acad. Nutr. Diet. 2015, 115, 1789-1797. [CrossRef] 